LUNFD6/(NFFL-7081)-Rev. 1994

hep-ph/9409327

\title{
Bose-Einstein Correlations for Longitudinally Expanding, Finite Systems
}

\author{
T. Csörgö 1,2 用 \\ ${ }^{1}$ KFKI Research Institute for Particle and Nuclear Physics of the \\ Hungarian Academy of Sciences, H-1525 Budapest 114, P.O. Box 49. Hungary \\ ${ }^{2}$ Department of Elementary Particle Physics, Physics Institute, \\ University of Lund, Sölvegatan 14, S-22362 Lund, Sweden
}

August 17, 1994

\begin{abstract}
Bose-Einstein correlations and momentum distributions are calculated for a longitudinally expanding boson source, where the expanding system has a finite size in space-time rapidity. Such systems are physically realized in any of the single jets in high energy $e^{+} e^{-}$, lepton-hadron and hadron-hadron collisions as well as in high energy heavy ion collisions, where the projectile is not really heavy. The $1 \mathrm{D}$ expansion generates a thermal length-scale in the longitudinal direction, which together with the finite length of the expanding tube effects both the momentum distribution and the Bose-Einstein correlation function. The Bose-Einstein correlations are shown to be more sensitive to the smaller, while the momentum distribution to the longer of the two longitudinal length-scales.
\end{abstract}

*E-mail: csorgo@sunserv.kfki.hu 
Introduction. There is a renewed interest in the study of relativistically expanding one dimensional systems partly due to the recent high energy heavy ion programme at CERN and AGS. The underlining space-time picture is thought to be determined by the Bose-Einstein correlation measurements. Both the Bose-Einstein correlations and the momentum-spectra were determined, see e. g. the contributions of the NA35 and NA44 collaborations in ref. [1]. In case of high energy elementary particle reactions where jets dominate the underlining space-time picture, usually only static parameterizations of the Bose-Einstein correlation functions (BECF-s) are used, with very few exceptions. For an introduction and review see ref. [2].

In this Letter the importance of the dynamical picture is emphasized. It seems to be necessary to analyze simultaneously the invariant momentum distribution (IMD) as well as the BECF-s in the longitudinally comoving system, LCMS, in order to measure the usually very long longitudinal size of the jets.

Particle interferometry for one dimensionally expanding hydrodynamical systems were studied first in ref. [3] where Bose-Einstein interferometry was considered for an infinite, longitudinally expanding Bjorken-tube. Kolehmainen and Gyulassy considered the onedimensionally expanding ideal inside-outside cascade [4], which was further elaborated in refs. [5]. The correlations in between rapidity and space-time rapidity were considered for 1D expanding strings in ref. [6] where also the relation of the sidewards and outward momentum components were clarified. However, in these works the rapidity distribution of the final particles remained somewhat arbitrary and there was only one characteristic length-scale in the longitudinal direction. This characteristic length-scale, the "length of homogeneity" or thermal length, appeared due to the interplay of the flow-gradient and the temperature in ref. [3] while it appeared as a characteristic rapidity - space-time rapidity correlation length in ref [6]. In this Letter the interplay between the finite size of the bosonemitting source together with the finite "thermal length" shall be considered in detail.

One-dimensionally expanding systems are thought to be realized by the hadronic strings in the case of 2-jet events in high energy elementary particle reactions. Any of the jets 
of high energy $p p, p \bar{p}, e^{+} e^{-}$or other $l+h$ collisions corresponds to an approximately onedimensionally expanding system. Boson interferometry for decaying hadronic strings was studied first in ref. [7] and later in details in ref. [8]. In these publications, however, the interplay between the total longitudinal length of the string and the BECF was not considered. High energy heavy ion collisions may also create one-dimensionally expanding systems, especially in the case of light projectiles. Heavier projectiles may create three-dimensionally expanding systems which shall not be discussed here.

Both the momentum spectra and the BECF are prescribed in the applied Wignerfunction formalism [9,2]. In this formalism the BECF is calculated from the two-body Wigner-function assuming chaotic particle emission. In the final expression the timederivative of the Wigner function is approximated [9,2] by a classical emission function $S(x ; p)$, which is the probability that a boson is produced at a given $x=(t, \vec{r})=(t, x, y, z)$ point in space-time with the four-momentum $p=(E, \vec{p})=\left(E, p_{x}, p_{y}, p_{z}\right)$. The particle is on the mass shell, $m^{2}=E^{2}-\vec{p}^{2}$, and the off-shell emission functions entering into the BECF [9] are approximated by the off-shell continuation of the on-shell emission functions. In this Letter the effect of final state Coulomb and Yukawa interactions shall be neglected.

The emission function. We model the emission function in terms of the longitudinally boost-invariant variables: the longitudinal proper-time, $\tau=\sqrt{t^{2}-z^{2}}$, the space-time rapidity $\eta=0.5 \ln \left(\frac{t+z}{t-z}\right)$, the transverse mass $m_{t}=\sqrt{E^{2}-p_{z}^{2}}$ and the momentum-space rapidity $\mathrm{y}=0.5 \ln \left(\frac{E+p_{z}}{E-p_{z}}\right)$. We shall assume that the emission function is characterized by a given space-time rapidity distribution $G(\eta)$, a distribution in the transverse production points $I\left(r_{t}\right)$ and a proper-time distribution $H(\tau)$. The correlations between rapidity and space-time rapidity are taken into account by the conditional probability $J_{m_{t}}(\eta-\mathrm{y})$, which gives the probability that a boson is emitted with rapidity y from the point-like source characterized by the space-time rapidity $\eta$. For a thermal emission, the width of this function is a function of the transverse mass $m_{t}$, as we shall see below. We may also introduce an arbi- 
trary $g\left(\vec{p}_{t}\right)$ transverse momentum distribution. Thus the emission function can be written in the following way:

$$
S\left(x ; \mathrm{y}, \vec{p}_{t}\right) d^{4} x=g\left(\vec{p}_{t}\right) G(\eta) H(\tau) I\left(r_{t}\right) J_{m_{t}}(\eta-\mathrm{y}) d \eta d \tau d^{2} \vec{r}_{t}
$$

First, we shall consider results for general, unspecified functional form of the spacetime distribution functions $G(\eta), H(\tau)$ and $I\left(r_{t}\right)$ and discuss how these quantities can be determined from the simultaneous analysis of the BECF and that of the IMD. We shall also apply Gaussian approximations for the distribution functions of $\tau, \eta, \eta-\mathrm{y}$ and $r_{t}$ as follows

$$
\begin{gathered}
G(\eta)=\frac{1}{\left(2 \pi \Delta \eta^{2}\right)^{1 / 2}} \exp \left(-\frac{\eta^{2}}{2 \Delta \eta^{2}}\right) \\
I\left(r_{t}\right)=\frac{1}{\left(2 \pi R_{G}^{2}\right)} \exp \left(-\frac{r_{t}^{2}}{2 R_{G}^{2}}\right) \\
H(\tau)=\frac{1}{\left(2 \pi \Delta \tau^{2}\right)^{1 / 2}} \exp \left(-\frac{\left(\tau-\tau_{0}\right)^{2}}{2 \Delta \tau^{2}}\right), \\
J_{m_{t}}(\eta-\mathrm{y})=\frac{1}{\left(2 \pi \Delta \eta_{T}^{2}\right)^{1 / 2}} \exp \left(-\frac{(\eta-\mathrm{y})^{2}}{2 \Delta \eta_{T}^{2}}\right) .
\end{gathered}
$$

This Gaussian ansatz results in a simple analytic parameterization of the BECF as well as for the rapidity-dependent part of the IMD. The transverse momentum dependent factor, $g\left(p_{t}\right)$ remains unspecified and cancels from the results.

It is to be emphasized that by introducing a finite width $\Delta \eta$ we break the boost-invariance of our source in the longitudinal direction too. Thus we expect a non-stationary rapidity distribution. The stationary rapidity distribution corresponds to the $\Delta \eta_{T} \rightarrow \infty$ limit.

In this model, the source at a given space-time - rapidity $\eta$ is emitting bosons with the rapidity $\mathrm{y}=\eta$, however with a non-vanishing width of the rapidity distribution of the emitted bosons. This width, $\Delta \eta_{T}$, can be determined by Monte-Carlo simulations in the case of decaying strings e.g. in the Lund model $\Delta \eta_{T} \approx 0.4$, refs. [12,6]. It can be calculated in the framework of hydrodynamics from the relativistic Boltzmann-distribution, 
$f(x ; p)=C \exp \left(-\frac{p \cdot u(x)}{T}\right)$, where the freeze-out temperature is denoted by $T$ and the fourvelocity of the $1 \mathrm{D}$ expanding matter at space-time point $x$ is given by the scaling Bjorken expansion [10] as

$$
u(x)=(t / \tau, 0,0, z / \tau)=(\cosh (\eta), 0,0, \sinh (\eta))
$$

Since the four momentum $p$ can be also written as $p=\left(m_{t} \cosh (\mathrm{y}), \vec{p}_{t}, m_{t} \sinh (\mathrm{y})\right)$, the relativistic Boltzmann distribution for the 1D expanding systems can be rewritten as

$$
f\left(x ; \mathrm{y}, p_{t}\right)=J_{m_{t}}(\eta-\mathrm{y})=C \exp \left(-\frac{m_{t} \cosh (\eta-\mathrm{y})}{T}\right) \approx C^{\prime} \exp \left(-\frac{m_{t}(\mathrm{y}-\eta)^{2}}{T}\right)
$$

which is the same form as in eq. 5. Thus in the case of the thermal emission, the width of the rapidity distribution of the particles is a decreasing function of the transverse mass,

$$
\Delta \eta_{T}\left(m_{t}\right)=\sqrt{\frac{T}{m_{t}}} .
$$

This completes the specification of the model.

Evaluation of the space-time integrals. We can evaluate the integrals in the LCMS, the longitudinally comoving system. Here we parameterize the mean momentum of the particles as $K=\left(K_{0}, K_{T, O}, 0,0\right)$ i.e. the longitudinal component of the mean momentum is vanishing. Since the mean momentum is purely transverse to the $z$ axis (jet or beam axis), it is pointing towards a direction which is named [11,6] the out direction. This we chose to coincide with our $x$ axis and index by $(T, O)$. The y axis shall be called the side component, indexed with $(T, S)$ (Transverse, Side ). In the Laboratory system the LCMS moves with the mean rapidity of the pair, $Y=\frac{1}{2} \ln \left(\frac{E_{1}+E_{2}+p_{z, 1}+p_{z, 2}}{E_{1}+E_{2}-p_{z, 1}-p_{z, 2}}\right)$. In the LCMS, we index the variables with ('). We calculate the auxiliary function $\tilde{S}(\Delta k, K)$ in the LCMS for small values of the components of the relative momentum $\Delta k=\left(\beta_{T} Q_{T, O}, Q_{T, O}, Q_{T, S}, Q_{L}\right)$. In the integration we utilize the following equalities or approximations: 


$$
\begin{aligned}
\eta^{\prime}=\eta-Y & \text { and } \quad \mathrm{y}^{\prime}=\mathrm{y}-Y \\
t^{\prime}=\tau \cosh \left(\eta^{\prime}\right) \approx \tau \quad \text { and } & z^{\prime}=\tau \sinh \left(\eta^{\prime}\right) \approx \tau \eta^{\prime} \approx \tau_{0} \eta^{\prime} .
\end{aligned}
$$

The latter two approximations are valid when the width of the rapidity distribution of the bosons emitted at a fixed value of $\eta^{\prime}$ is smaller than unity, $\Delta \eta_{T}<1$, and when the width of the proper-time distribution is smaller than the mean proper-time, $\Delta \tau<\tau_{0}$. The first approximation is valid for thermal emission in the $m_{t}$ region $T<m_{t}$ as well as e.g for the Lund strings $\left(\Delta \eta_{T} \approx 0.4\right)$. (Note, that in case of a decaying hadronic string, the fluctuations of the break-up points create the rapidity - space-time rapidity correlation length $\Delta \eta_{T}$.)

The second approximation is more safe for the case of heavy ion collisions, where the mean freeze-out time is about $\tau_{0} \approx 4-5 \mathrm{fm} / \mathrm{c}$, and the estimated duration is very short, $\Delta \tau<2 \mathrm{fm} / \mathrm{c}, \mathbb{1}$. With the help of the above approximations the integrals can be evaluated.

General results. The one-particle IMD can be calculated for arbitrary space-time distribution functions as under the assumption that the relativistic Boltzmann distribution creates the correlations in between rapidity and space-time rapidity, eq. 7.

$$
\begin{aligned}
\frac{d^{2} n}{d \mathrm{y} d m_{t}^{2}} & =g\left(p_{t}\right) \int_{-\infty}^{\infty} d \eta G(\eta) J_{m_{t}}(\eta-\mathrm{y}), \\
G(\mathrm{y}) & =\lim _{m_{t} \rightarrow \infty} \frac{1}{g\left(\vec{p}_{t}\right)} \frac{d^{2} n}{d \mathrm{y} d m_{t}^{2}},
\end{aligned}
$$

Based on the above formulas, the function $G(\eta)$ can be measured as the asymptotic (large $\left.m_{t}\right)$ limit of the rapidity dependent part of the IMD $d^{2} / d y / d m_{t}^{2}$. Then the measured $G(\eta)$ function can be convoluted with the relativistic Boltzmann distribution according to equations 17, 11, 12. Thus the $m_{t}$ dependent width in rapidity of the IMD is prescribed from the model and can be contrasted to the data for a general shape of the $G(\eta)$ distribution. This rapidity dependent part of the IMD is obtained as a thermally broadened space-time rapidity distribution, and the asymptotic, large $m_{t}$ limit of the rapidity distribution corresponds to the space-time rapidity distribution. With decreasing $m_{t}$, the thermal broadening becomes larger and larger except the cases when $i$, the rapidity plateau becomes infinitely 
long, or when ii, the decreasing part of the rapidity distribution falls outside the experimental acceptance.

The general result for BECF is given in the LCMS of the particle pairs as

$$
C(\Delta k, K)=1+\left|\tilde{H}\left(\beta_{T} Q_{T, O}\right)\right|^{2}\left|\tilde{I}\left(Q_{T, O}, Q_{T, S}\right)\right|^{2}\left|\tilde{G}_{Y, M_{t}}^{e f f}\left(\tau_{0} Q_{L}\right)\right|^{2},
$$

Here $\tilde{H}$ etc. denotes the Fourier-transformed space-time distribution and the effective spacetime rapidity region where the boson pairs are emitted from is denoted by

$$
G_{Y, M_{t}}^{e f f}(\eta)=\frac{1}{\int d \eta G(\eta) J_{M_{t}}(\eta-Y)} G(\eta) J_{M_{t}}(\eta-Y)
$$

and the mean transverse mass is denoted by $M_{t}=\left(m_{t, 1}+m_{t, 2}\right) / 2$. Thus in the one dimensionally expanding case the interpretation of the components of the correlation function is the following: the duration in proper-time gives contribution to the out component of the correlation function while the transverse distribution of the emission points, $I\left(\vec{r}_{t}\right)$ yields a symmetric contribution to both the out and the side component. The longitudinal component measures that region in the longitudinal direction where bosons with similar momenta may emerge from.

For an arbitrary distribution of rapidities, e.g. a flat central plateau plus some wings, these results give an opportunity for a measurement of the underlying, non-Gaussian spacetime distribution.

Results for the Gaussian ansatz. The general results may be further simplified in the framework of the Gaussian approximations, 207 as follows:

$$
\frac{d^{2} n}{d y d m_{t}^{2}}=\frac{1}{\left(2 \pi \Delta y^{2}\left(m_{t}\right)\right)^{1 / 2}} g\left(p_{t}\right) \exp \left(-\frac{y^{2}}{2 \Delta y^{2}\left(m_{t}\right)}\right)
$$

and

$$
C(\Delta k, K)=1+\exp \left(-\left(R_{T, S}^{2} Q_{T, S}^{2}+R_{T, O}^{2} Q_{T, O}^{2}+R_{L}^{2} Q_{L}^{2}\right)\right)
$$

where the parameters are related to those of the source by 


$$
\begin{aligned}
\Delta y^{2}\left(m_{t}\right) & =\Delta \eta^{2}+\Delta \eta_{T}^{2}\left(m_{t}\right), \\
R_{T, S}^{2} & =R_{G}^{2}, \\
R_{T, O}^{2} & =R_{G}^{2}+\beta_{T}^{2} \Delta \tau^{2}, \\
R_{L}^{2}\left(m_{t}\right) & =\tau_{0}^{2} \Delta \eta_{*}^{2}\left(m_{t}\right) .
\end{aligned}
$$

The quantity $\Delta \eta_{*}$ corresponds to the effective correlation length between rapidity and spacetime rapidity, as seen by the BECF. Its square is half of the harmonic mean of the squares of the thermal correlation length and the total size of the pseudo-rapidity distribution,

$$
\frac{1}{\Delta \eta_{*}^{2}\left(m_{t}\right)}=\frac{1}{\Delta \eta^{2}}+\frac{1}{\Delta \eta_{T}^{2}\left(m_{t}\right)}
$$

thus it is always dominated by the shorter of the thermal and geometrical length scales ( $\Delta \eta_{T}$ and $\Delta \eta$, respectively). However, the square of the width of the rapidity distribution, $\Delta y^{2}\left(m_{t}\right)$ is the quadratic sum of the geometrical and the thermal length scales, thus it is dominated by the longer of the two.

Discussion. For thermally emitting sources the Boltzmann distribution predicts a very specific change of the rapidity-width of the $d^{2} / d y / d m_{t}^{2}$ distribution as a function of the transverse mass:

$$
\Delta y^{2}\left(m_{t}\right)=\Delta \eta^{2}+\frac{T}{m_{t}} .
$$

This effect, based on simple kinematics, can be experimentally checked by measuring the $y$ - width of the IMD-s at different values of fixed $m_{t}$. Fitting the result with the above equation, both the geometrical size of the emission in pseudo-rapidity, $\Delta \eta$ and the freeze-out temperature $T$ can be extracted.

The transverse mass dependence of the width of the rapidity distribution becomes especially well measurable in the intermediate region, where the thermal and geometrical length-scales are similar, see Fig. 1 and Fig. 2. as examples.

In principle, it is possible to determine the same set of parameters from a measurement of the $m_{t}$ dependence of the $R_{L}$ parameter of the BECF-s as follows: 


$$
R_{L}^{2}\left(m_{t}\right)=\tau_{0}^{2} \frac{T}{m_{t}} \frac{1}{1+\frac{T}{m_{t} \Delta \eta^{2}}} .
$$

This provides a possibility for the consistency check when the model is compared with the data. Note that this expression coincides with Sinyukov's formula for $\Delta \eta \rightarrow \infty$, as it is expected since in that limit our model source corresponds to the infinite expanding Bjorkentube. Thus the above expression can be considered as the generalization of Sinyukov's formula for finite, $1 \mathrm{D}$ expanding systems. At present energies, we expect that the corrections are significant, The effects of finite geometrical size on the longitudinal radius parameter are visualized on Fig.3. and Fig.4. In both cases the mean freeze-out time was chosen to be $\tau_{0}=7.0 \mathrm{fm} / \mathrm{c}$. For $\Delta \eta=0.5$ the correction at low $m_{t}$ yields about a factor of 0.5 decrease in the longitudinal radius, because the finite geometrical size is comparable with the thermal length-scale for moderate values of $m_{t}$ in the case of Fig. 3. For a larger system with $\Delta \eta=1.0$ the finite size correction factor is about 0.8. The difference between Sinyukov's formula and its finite-size corrected version vanishes for high values of the transverse mass and also decreases for increasing values of the geometrical size $\Delta \eta$.

The above expression is a function of three free parameters, $\tau_{0}, T$ and $\Delta \eta$. To determine all the three parameters simultaneously from the $m_{t}$ dependence of the BECF one needs very dedicated set-up. It seems to be more straightforward to determine the $T$ and $\Delta \eta$ parameters from the $m_{t}$ dependence of the rapidity - width of the $d^{2} n / d y / d m_{t}^{2}$ distribution, and use the interferometry measurement to infer the value of the mean freeze-out time $\tau_{0}$. Thus the importance of dedicated Bose-Einstein experiments and wide acceptance detectors is emphasized simultaneously: the BECF has to be studied as a function of the $m_{t}$ and the $\mathrm{y}$-width of the IMD has to be determined as a function of $m_{t}$, too.

In the considered case, where the transverse position and the transverse momentum distribution decouple, the side component of the BECF measures the geometrical size, and the out component measures the quadratic sum of both the geometrical radius and the width 
of the proper-time distribution times the mean transverse velocity of the pair. The latter can be considered as an increase of the effective source size in the out direction due to the evaporation and the mean speed of the bosons.

Limiting Cases. Depending on the relative size of the thermal and the geometrical logitudinal scales, $\Delta \eta_{T}$ and $\Delta \eta$, we have two limiting cases: Case $i$, If the geometrical size becomes much larger than the thermal length, the rapidity-width of the IMD approaches the geometrical size and becomes independent of the transverse mass,

$$
\begin{aligned}
\frac{d^{2} n}{d y d m_{t}^{2}} & \propto \exp \left(-\frac{y^{2}}{2 \Delta \eta^{2}}\right) \quad \text { if } \quad \Delta \eta>>\Delta \eta_{T}, \\
R_{L}^{2} & =\tau_{0}^{2} \Delta \eta_{T}^{2}=\tau_{0}^{2} \frac{T}{m_{t}} \quad \text { if } \quad \Delta \eta>>\Delta \eta_{T} .
\end{aligned}
$$

In this limit, corresponding to long and relatively cold expanding system, the geometrical longitudinal size is dominating the width of the rapidity distribution, while the thermal longitudinal length-scale is dominating the longitudinal radius parameter of the BECF, measured in the LCMS of the boson pair.

Case $i$, If the rapidity distribution of the particles at a fixed space-time rapidity is much more wider than the geometrical length-scale, then interferometry measures the geometrical size properly, and the temperature can be inferred from the width of the rapidity distribution:

$$
\begin{aligned}
\frac{d^{2} n}{d y d m_{t}^{2}} & \propto \exp \left(-\frac{y^{2}}{2 \Delta \eta_{T}^{2}}\right) \propto \exp \left(-\frac{E}{T}\right) \quad \text { if } \quad \Delta \eta_{T}>>\Delta \eta \\
R_{L}^{2} & =\tau_{0}^{2} \Delta \eta \quad \text { if } \quad \Delta \eta>>\Delta \eta_{T} .
\end{aligned}
$$

This limiting case corresponds to a relatively hot and small, expanding system. The effects of the expansion cancel from the results here. The expressions are the same as those for a hot source with no expansion.

Note that this work is an application of the ideas presented in [13] to the case of the relativistic one dimensionally expanding systems. 
In summary, we have calculated the rapidity distribution and the Bose-Einstein correlation function in the longitudinally comoving frame for one-dimensionally expanding systems. In the longitudinal direction, there are two length-scales present: the geometrical and the thermal one. The latter is either due to the combined effect of the flow-gradient and the temperature or e.g. to the stochastic nature of the string fragmentation. In an analytically tractable model for the emission function, the longitudinal component of the Bose-Einstein correlation function is shown to measure dominantly the shorter while the width of the rapidity distribution measures dominantly the longer length-scale. This confers the intuitive picture that the strings are really long, but cannot be seen by BEC measurements due to their expansion. The geometrical size can be determined from the $m_{t}$ dependence of the rapidity-width of the one-particle invariant momentum distribution. The freeze-out temperature, the mean proper-time, the width of the proper-time distribution as well as the geometrical size in the transverse direction can be determined within this picture by a combined use of the radius parameters in the correlation function on one hand and by the analysis of the transverse mass dependent rapidity-width of the invariant momentum distribution on the other hand.

Acknowledgments: I would like to thank B. Lörstad for kind hospitality and stimulation during my stay at University of Lund. I thank G. Gustafson and U. Heinz for helpful discussions and thank to Á. Till and L. P. Csernai for kind hospitality at the University of Bergen in March 1994. This work was supported in part by a NORFA grant, by the NFR and the Hungarian Academy of Sciences exchange grant, by the Human Capital and Mobility (COST) programme of the European Economic Community under grants No. CIPA - CT 92 - 0418 (DG 12 HSMU), by the Hungarian NSF under Grant No. OTKA-F4019, and also as a prelude it is supported by the Hungarian - U. S. Joint Fund. 


\section{REFERENCES}

[1] Proceedings of the Quark Matter conferences, especially Nucl. Phys. A498, (1989), Nucl. Phys. A525, (1991), Nucl. Phys. A544, (1992) and Nucl. Phys. A566, (1993).

[2] B. Lörstad, Int. J. Mod. Phys. A12 (1989) 2861-2896.

[3] A. Makhlin and Y. Sinyukov, Z. Phys. C39, 69 (1988). (1986) 203.

[4] K. Kolehmainen, M. Gyulassy, Phys. Lett. B180

[5] S. S. Padula and M. Gyulassy, Nucl. Phys.B339 378 (1990)

[6] T. Csörgő and S. Pratt, KFKI-1991-28/A, p. 75.

[7] B. Andersson and W. Hoffman, Phys. Lett. B169 (1986) 364

[8] M. G. Bowler, Particle World 2 (1991) 1.-6.

[9] S. Pratt, T. Csörgő, J. Zimányi, Phys. Rev. C42 (1990) 2646; S. Pratt, Phys. Rev. D 33, 1314 (1986).

[10] J. D. Bjorken, Phys. Rev. D27, (1983) 140.

[11] G. F. Bertsch, Nucl. Phys. A498 (1989) 173c

[12] B. Andersson et al, Phys. Rep. 97 (1983) 33

[13] T. Csörgő, B. Lörstad and J. Zimányi, "Quantum Statistical Correlations for Slowly Expanding Systems", preprint LUNFD6/(NFFL-7084) 1994, Physics Letters B in press. 


\section{FIGURES}

Fig. 1.

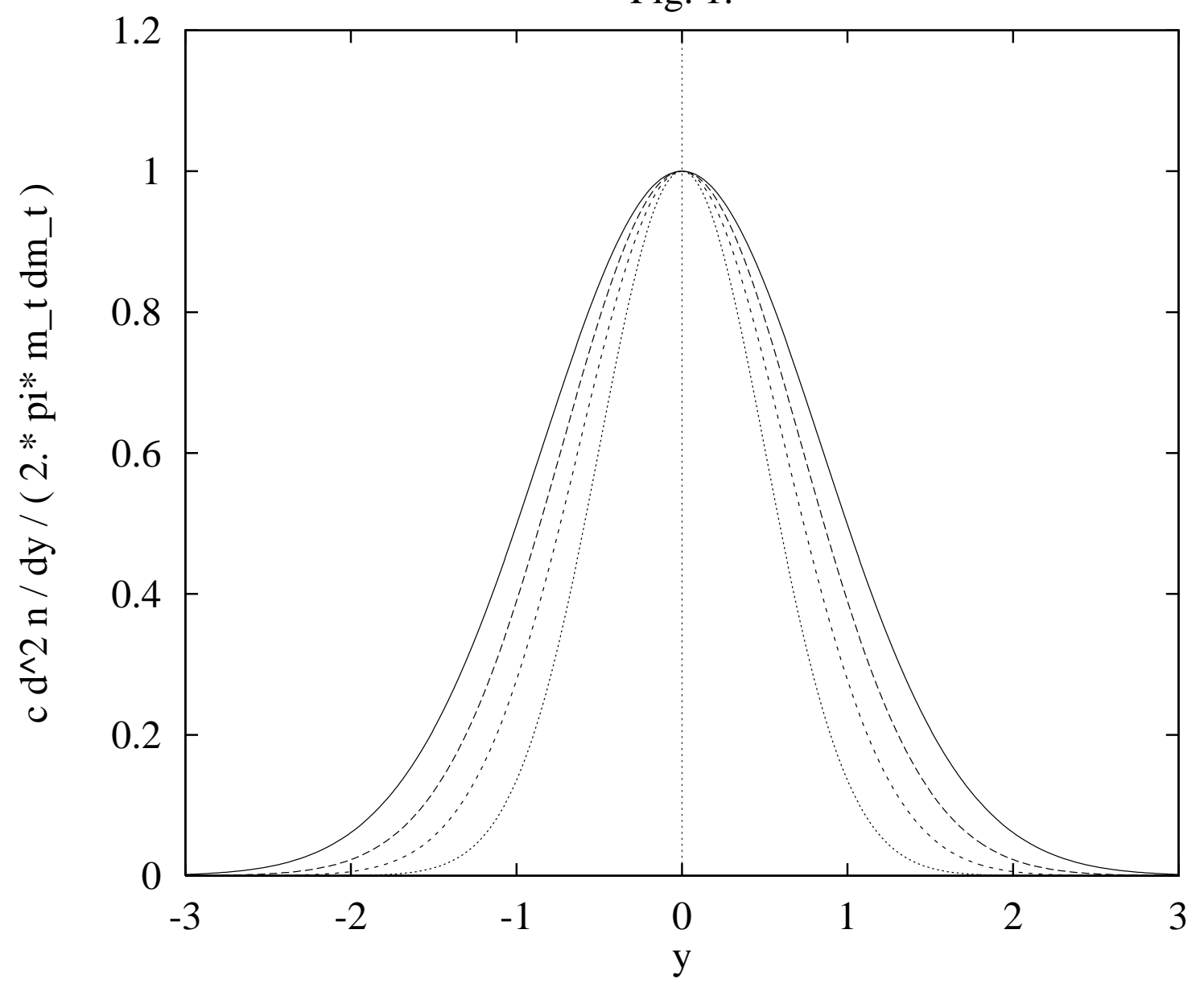

FIG. 1. The change of the rapidity-width of the $d^{2} n / d y / d m_{t}^{2}$ distribution for increasing values of the (fixed) transverse mass, $m_{t}=0.3,0.5,1.0 \mathrm{GeV} / \mathrm{c}$, as shown by the solid, long-dashed and short-dashed lines, respectively. The dotted line stands for the asymptotic $m_{t} \rightarrow \infty$ limit, coinciding with $G(y)$, the geometrical shape of the source in the longitudinal direction which was chosen to be a Gaussian with $\Delta \eta=0.5$. The distributions were normalized to the same maximum value of 1 . The pion mass $(m=0.14 \mathrm{GeV})$ and a freeze-out temperature of $T=0.14 \mathrm{GeV}$ was utilized. 
Fig. 2.

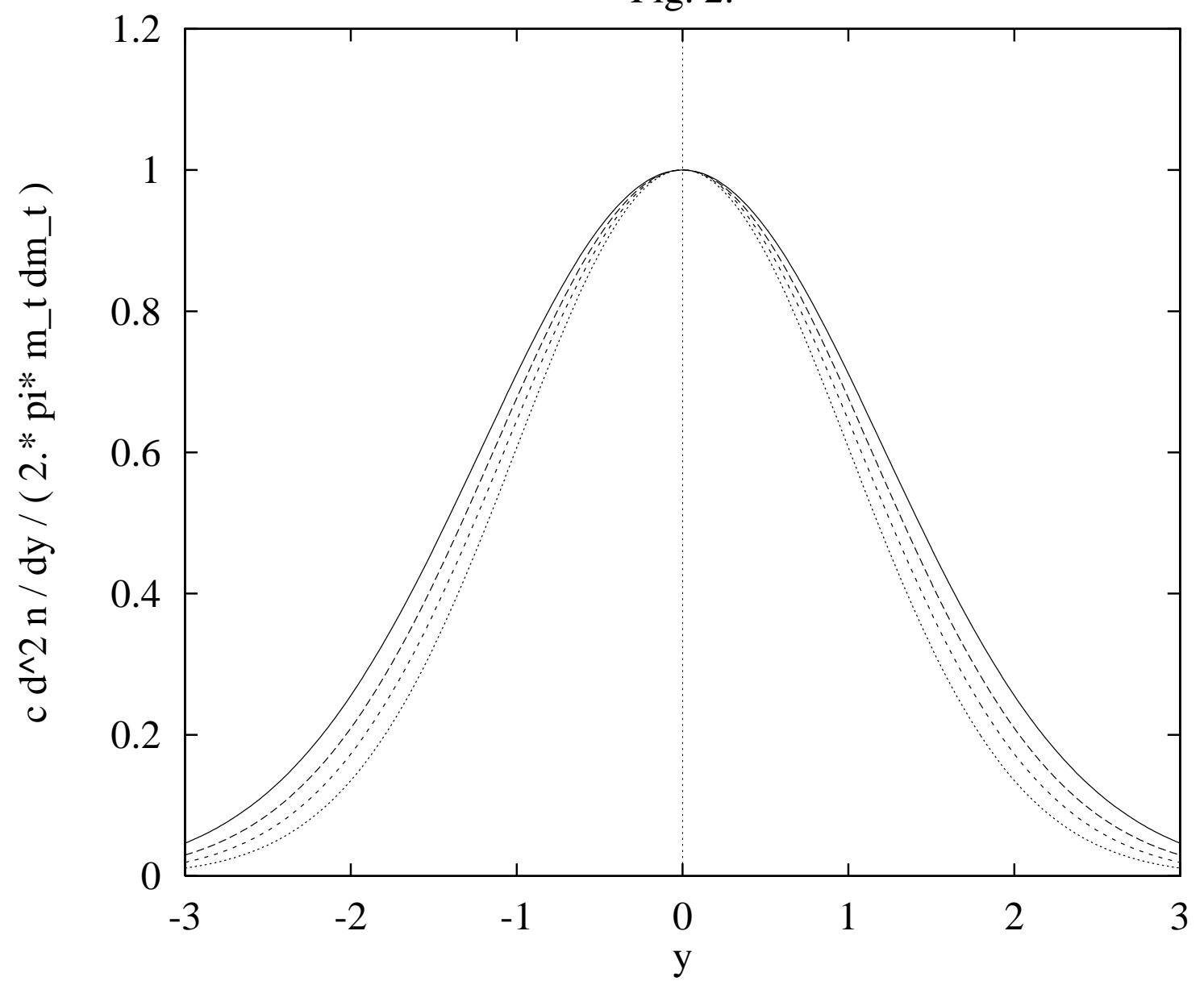

FIG. 2. Same as Fig. 1. but for $\Delta \eta=1.0$. 
Fig. 3.

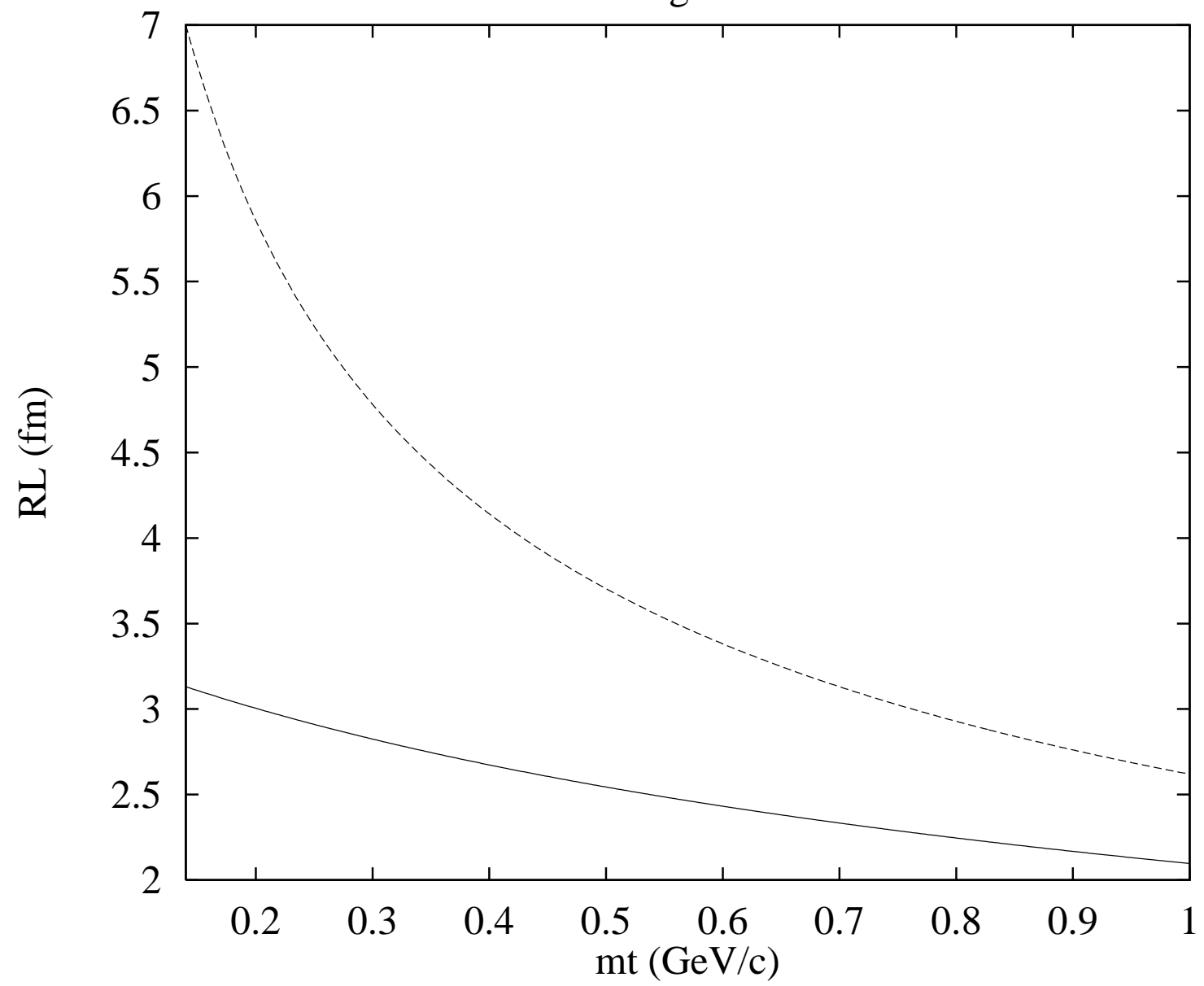

FIG. 3. The $m_{t}$ dependence of the $R_{L}$ radius parameter for finite $1 \mathrm{D}$ expanding systems is shown by the solid curve. The mean freeze-out time is chosen to be $t_{0}=7 \mathrm{fm} / \mathrm{c}$ and $\Delta \eta=0.5$. The other parameters are the same as for Fig. 1. Dashed line shows the prediction for $R_{L}$ based on Sinyukov's formula. 
Fig. 4.

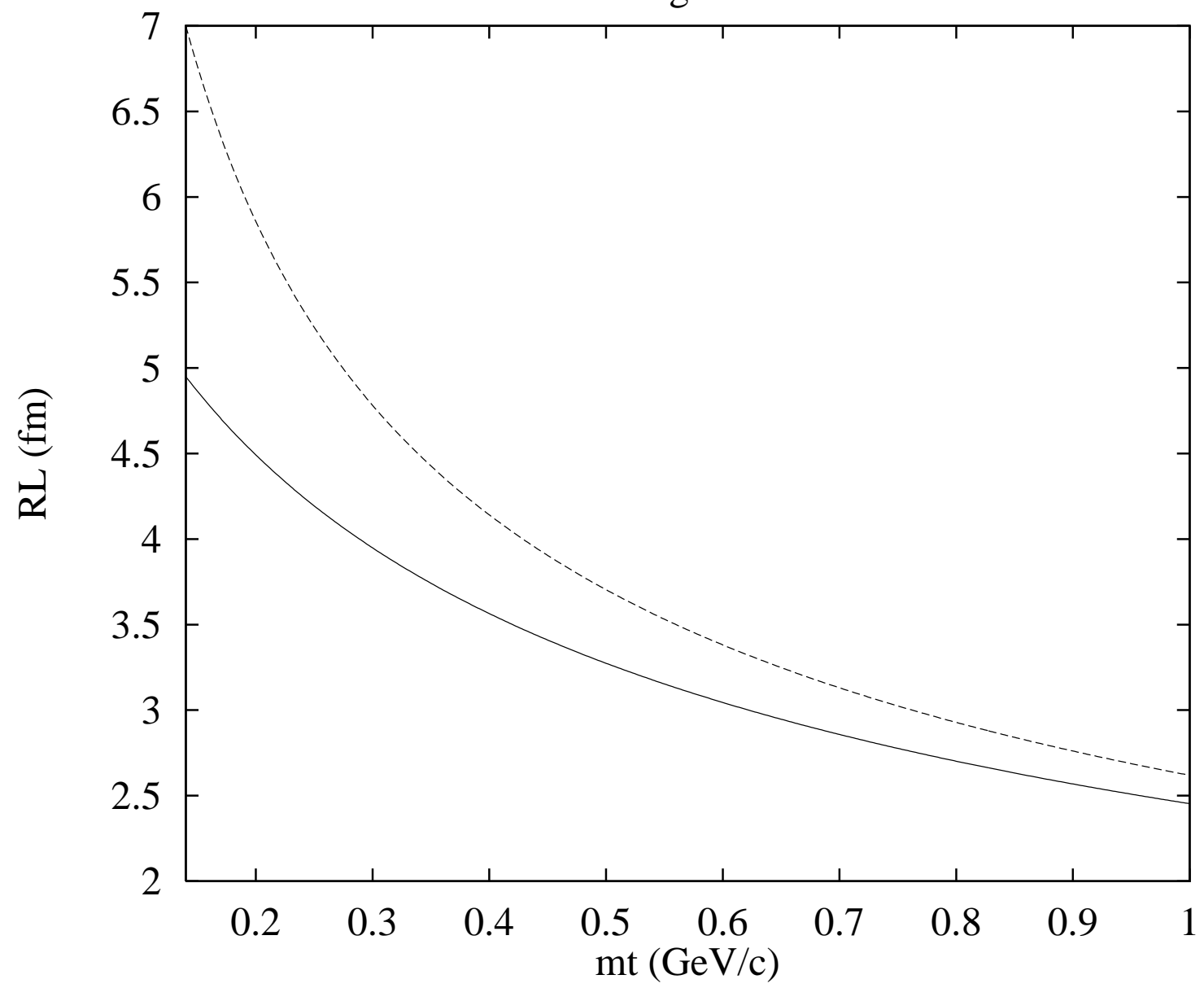

FIG. 4. Same as Fig. 3. but for $\Delta \eta=1.0$. 